\title{
Modeling of Multi-groups Solid Tumor Growth Based on Tumor Biochemical Environment
}

\author{
Lujun Zhang, ${ }^{1, \mathrm{a}}$, Kai Han ${ }^{1, \mathrm{~b}}$ and Yonggui $\mathrm{Xu}^{1, \mathrm{c}, *}$ \\ ${ }^{1}$ School of Physics and Optoelectronic Engineering, Weifang University, Weifang 261061, China \\ azhang_lujun1985@163.com, bhankai@wfu.edu.cn, 'yongguixu@163.com
}

Keywords: Solid tumor growth, mathematical modeling, diffusion-convection-reaction equation, tumor biochemical environment.

\begin{abstract}
With the aid of computer technology and the use of mathematical physical method, it is of special significance to construct a tumor growth model, to obtain the knowledge of tumor biology behaviors and to assistant clinical treatment. Based on the solid tumor biochemical environment, the proposed model focused on three factors: the concentration of nutrient, extracellular matrix giant molecules, and also tumor surrounding tissue environment characteristics, which were combined to diffusion terms, convection terms and reaction terms of a diffusion-convection-reaction equation which was the foundation of the model. The tumor cells were divided into two groups of proliferating cells and necrotic cells. The proposed model established a coupling model which had respective equations for these two groups of tumor cells, to show the dynamic tumor growth process with the interaction effect of these two groups. The simulation results demonstrated that the proposed model could show the process of tumor growth, and the interaction between the tumor growth and the various factors, and also coincided with the actual tumor biological behaviors.
\end{abstract}

\section{Introduction}

In recent decades, a large number of biomedical engineering scientific workers dedicated to the use of mathematical models to study the mechanism of tumor growth. In all these tumor growth models, some were continuous models, which mainly focused on the large scale development of the whole tumor, considering the interaction between tumor cells and one or more chemicals to describe tumor cell population growth. This kind of models tended to regard tumor as a continuous system, rather than a combination of discrete cells, mainly reflecting the behavior of the overall process of tumor [1]. Some researchers thought that the continuous model over-focused on the overall process, ignoring the small scale cells, molecules and even gene level details [2]. Then they focused on establishment of discrete model [2, 3] or discrete-continuous model (hybrid model) [4, 5]. Anderson et al. [6, 7] were working on the hybrid model, using continuous equations to describe the concentration of various biochemical factors, both integrating discrete model to describe the growth movement of tumor cells, with considering all possible conditions of individual cells in the migration process. Wise et al. [8, 9] directly expressed cell proliferation, necrosis, and cell-cell adhesion factors by mathematical formulas, making it become a unified continuous model.

At present these common tumor growth models considers more and more rich levels, which can reflect more comprehensive, more detailed form of tumor growth. However, the concern is that if the cell number is great, then the calculation of discrete / hybrid model will be very large [7]. More importantly, many factors of cell or even smaller scales are of strong randomness, and some of its biological mechanism and behavior are even not clear. Because of the uncertainty of the small scale, the modeling process often requires to make many assumptions and rules for each cell movement, which leads to the lack of practical significance.

This paper proposes a solid tumor mathematical model mainly from the large scale, with the small scale factors reflected in the large scale. The model uses the diffusion-convection-reaction equation as the foundation to simulate the overall growth of the solid tumor. Many biochemical environment 
factors are considered in this model, including nutrient concentration which directly determines the tumor cell proliferation rate [10], extracellular matrix (ECM) large molecules concentration which induces tumor cell chemotactic movement [7], and the tumor tissue environment. These factors have clear biological mechanism, and can be obtained from the medical image or medical experience data, easily parameterized. Furthermore, the tumor cells are divided into two groups (proliferating cells and necrotic cells) in this model, which results in an establishment of mutual restraint, dynamic growth of multi-groups solid tumor growth model.

\section{Basic Model of Solid Tumor Growth}

In addition to the blood, bone marrow and lymphatic system, tumors in other areas are collectively referred to as solid tumors. The experimental data and theoretical research showed that tumor originated from the interaction between tumor microenvironment and tumor cell genome, thus tumor microenvironment, composed of the biochemical environment around tumor cells, plays the important role in the process of tumor formation and growth [10].

This paper uses the diffusion-convection-reaction equation and the diffusion-reaction equation as the basic model of solid tumor growth, with considering the effects of two important biochemical environmental factors: nutrient and extracellular matrix (ECM):

$$
\begin{aligned}
\frac{\partial \varphi_{t}}{\partial t} & =\nabla \cdot\left(D_{t} \nabla \varphi_{t}\right)-\alpha \nabla \bullet\left(\varphi_{t} \nabla e\right)+\lambda_{p} n \varphi_{t} \\
\frac{\partial e}{\partial t} & =-\gamma_{e} \varphi_{t} e \\
\frac{\partial n}{\partial t} & =\nabla \bullet\left(D_{n} \nabla n\right)-\gamma_{n} \varphi_{t} n
\end{aligned}
$$

In this model, $\varphi_{t}$ represents the volume of the tumor, which will be normalized in the simulation, thus the location of the tumor can be judged by the size of volume. For example, the proportion of tumor cells volume here is greater than $50 \%$, then the tumor could be considered have grown into this position.

In formula 1, the right-hand side respectively represents diffusion term, convection term and reaction term. The diffusion term is used to express tumor cells outward invasion process, in which the diffusion coefficient $D_{t}$ means the rate of tumor cells invasion.

The convection term is used to represent the chemotactic migration movement of tumor cells. In the process of tumor growth, besides the invasion, the chemotactic migration, which is induced by the interactions between tumor cell and ECM (cell extracellular matrix, mainly composed of large molecules such as fibronectin, laminin, collagen) [7], also plays a key role. Thus $e$ means the concentration of ECM large molecules, then the velocity of the chemotactic migration is expressed as $\vec{v}=\alpha \nabla e$, where $\alpha$ is an adjustment factor, which indicates the degree of the concentration gradient. Due to that the tumor cells also secrete matrix degrading enzymes to degrade ECM, the formula 2 expresses the change of nutrient concentration $e$, in which $\gamma_{e}$ means the ECM degradation rate by tumor cells.

The reaction term is used to represent tumor cell proliferation which must be bound by the nutrition environment. Thus $n$ means the concentration of nutrient, $\lambda_{p}$ means tumor cell proliferation rate. The formula 3 uses the diffusion-reaction equation to express the change of nutrient concentration, in which $D_{n}$ means nutrient diffusion rate, and $\gamma_{n}$ means the rate of nutrient consumption by tumor cells. 


\section{Improved Tumor Growth Model}

\subsection{Improved Model with Tissue Environment}

In fact, the growth of tumor is not in a blank environment, but the different surrounding tissue environment will bring different pressure to tumor growth, some of which are easy to be invaded, while some of which may strongly resist tumor continuing to grow. In order to reflect the effect of surrounding tissue environment on tumor growth, this paper proposes an improved model by adding an environment parameter $\theta$ to characterize the properties of tissue environment. This parameter will be quantified in the range of $0 \sim 1$, and the closer to 1 indicates that the environment is easier to be invaded. Thus the formula 1 in basic model is modified as follow:

$$
\frac{\partial \varphi_{t}}{\partial t}=\nabla \bullet\left(\theta D_{t} \nabla \varphi_{t}\right)-\alpha \nabla \bullet\left(\varphi_{t} \theta \nabla e\right)+\theta \lambda_{p} n \varphi_{t}
$$

As it shown, the invasion rate, chemotactic migration velocity and proliferation rate are respectively modified as $\theta D_{t}, \alpha \theta \nabla e$ and $\theta \lambda_{p}$, positively proportional to $\theta$, which reflects the invasion degree of surrounding tissue environment.

\subsection{Improved Model with Necrotic Cells Group}

When tumor grows to a certain stage, tumor consumes a large amount of nutrients, thus the diffusion of nutrients will not meet the needs of tumor cells for further growth, and then the necrosis region in the tumor center will emerge [11]. Then the tumor cells are divided into proliferative and necrotic cells. The proliferative cells will continue to proliferate and to consume nutrients, while the necrotic cells will increase as the concentration of nutrients continues to decline, but will no longer consume nutrients. Based on this analysis, we improve the model by adding necrotic tumor cells group, the volume of which is expressed as $\varphi_{d}$. Then $\varphi_{t}-\varphi_{d}$ represents the volume of proliferative tumor cells. The final improved tumor growth model is as follows:

$$
\begin{aligned}
& \frac{\partial \varphi_{t}}{\partial t}=\nabla \cdot\left(D_{t} \theta \nabla \varphi_{t}\right)-\alpha \nabla \bullet\left(\varphi_{t} \theta \nabla e\right)+\theta \lambda_{p} n\left(\varphi_{t}-\varphi_{d}\right) \\
& \frac{\partial \varphi_{d}}{\partial t}=\nabla \cdot\left(D_{d} \theta \nabla \varphi_{d}\right)-\alpha \nabla \bullet\left(\varphi_{d} \theta \nabla e\right)+\lambda_{d} \cdot H\left(n_{d}-n\right) \cdot\left(\varphi_{t}-\varphi_{d}\right) \\
& \frac{\partial n}{\partial t}=\nabla \cdot\left(D_{n} \nabla n\right)-\gamma_{n}\left(\varphi_{t}-\varphi_{d}\right) n \\
& \frac{\partial e}{\partial t}=-\gamma_{e} \varphi_{t} e
\end{aligned}
$$

Formula 6 is the diffusion-convection-reaction equation of the necrotic cells, in which the diffusion and convection terms are consistent with the whole tumor. In reaction term, $\lambda_{d}$ means the rate of proliferative cells turning to the necrotic cells, and $n_{d}$ means the lower limit of nutrients concentration for proliferative cells surviving. The function $H(\bullet)$ is Heaviside function:

$$
H(\bullet)=\left\{\begin{array}{ll}
1 & \bullet \geq 0 \\
0 & \bullet<0
\end{array} \text { or } H(\bullet)=\frac{1}{2}\left(1+\frac{2}{\pi} \arctan \left(\frac{\bullet}{\varepsilon}\right)\right)\right.
$$

Then the reaction term in formula 6 indicates that once the nutrient concentration is lower than $n_{d}$, the proliferative cell will change to necrosis cell.

\section{Matlab Simulation and Result Analysis}

Table 1 Model Parameters

\begin{tabular}{|c|c|c|c|c|c|c|c|c|}
\hline$D_{t}$ & $D_{d}$ & $D_{n}$ & $n_{d}$ & $\gamma_{n}$ & $\gamma_{e}$ & $\lambda_{p}$ & $\lambda_{d}$ & $\alpha$ \\
\hline 0.5 & 0.5 & 1 & 0.4 & 0.3 & 0.1 & 0.6 & 0.1 & 0.3 \\
\hline
\end{tabular}


Matlab is used to simulate the proposed tumor growth model. This paper uses a square with $128 \times 128$ grid points as the calculation area. This paper respectively uses different shapes as the initial region of the tumor ( $\varphi_{t}=1$ in internal region, $\varphi_{t}=0$ in external region) to simulate the model, other parameters (dimensionless) are listed in Table 1.

The simulation is started with a regular round shape as the initial tumor region and a completely uniform biochemical environment, that the tumor surrounding tissue environment is identical, and nutrient concentration distribution and ECM concentration distribution are completely uniform ( $\theta=1, n=1, e=1$ in all region). The simulation results are shown in Fig. 1. It can be seen from the figure, in a completely uniform biochemical environment, the whole tumor (red line) also showes a completely uniform growth trend, and with the continuous decrease of nutrient concentration in the central region, the tumor appears necrotic region (blue line), which enlarges with increased time. This simple simulation results show that the proposed model can reflect the basic tumor invasion growth trend, and can reflect the tumor in the growth process will divide into two groups: proliferative cells (the area between the red line and blue line) and necrotic cells.

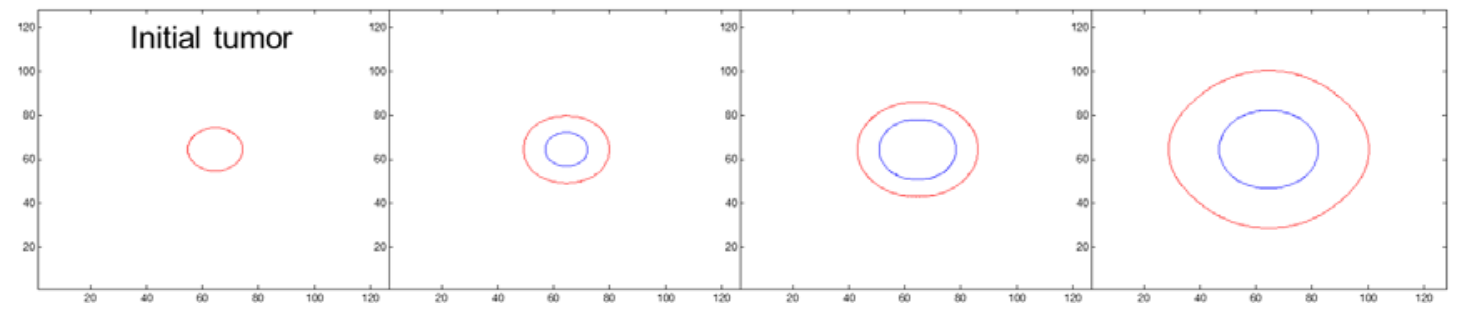

Fig. 1 Simulation results with regular initial tumor shape and uniform biochemical environment

Still with round initial tumor shape, the heterogeneous surrounding tissue environment is also simulated by initializing nonuniform parameter $\theta$. In the simulation, two regions with different degrees for tumor invasion are initially set up ( $\theta=0.01, \theta=0.4$ respectively). The initial tissue environment distribution and the tumor growth results are shown in Fig. 2 . The results show that the original regular tumor gradually becomes irregular: when tumor growth meets a hard-invaded tissue, the invasion and growth will lag behind other regions, especially when the tissue is too dense to tumor invasion, tumor growth will be forced to stay in the area outside and make a detour. This is in line with the actual tumor growth.

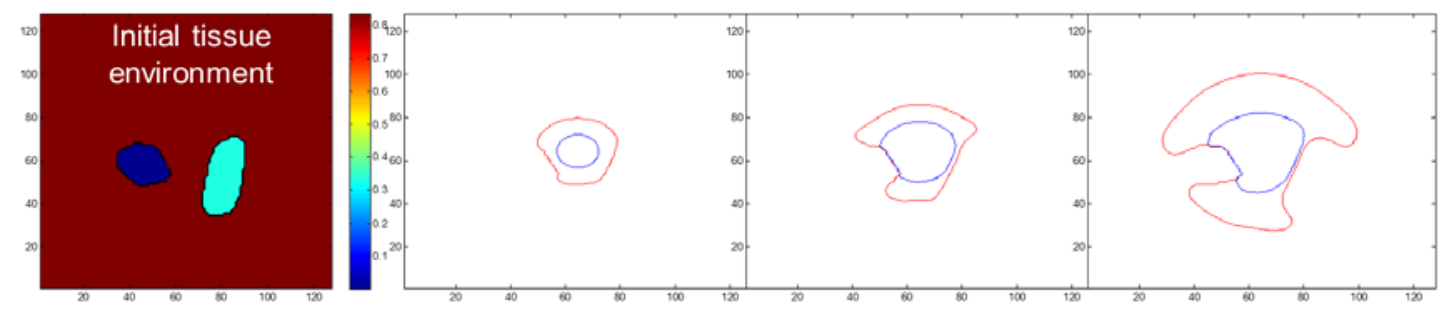

Fig. 2 Simulation results with regular initial tumor shape and nonuniform tissue environment

Finally, for an irregular initial tumor shape, and in different tissue environments (uniform or nonuniform), simulation results are shown in Fig. 3. It can be seen from the figure, tumor growth is affected by the tissue environment, but due to the irregular shape of the original tumor, the tumor will have specific shape itself in the growth process, and more easily grow toward the direction of protuberances. This simulation results are also consistent with the characteristics of tumor growth. Because tumor has to constantly absorb nutrients from the outside tissue, so in the process of tumor growth, keeping a shape with protuberances, which makes the tumor have more surface area exposed to facilitate the absorption of nutrients while the tumor volume increased, is a kind of tumor mechanism of self-protection [11]. 


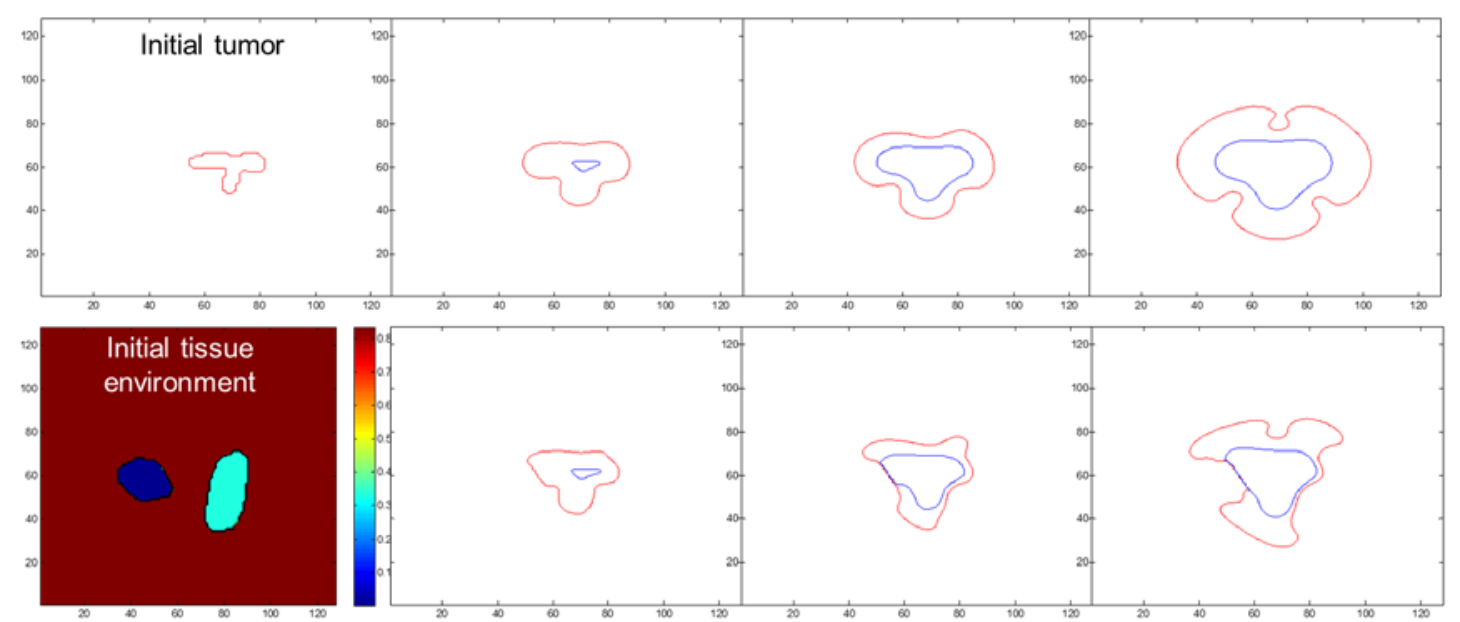

Fig. 3 Simulation results with irregular initial tumor shape and different tissue environments (top row: uniform tissue environment; bottom row: nonuniform tissue environment)

\section{Conclusions}

This paper mainly considered three factors of biochemical environment of solid tumor: the nutrient concentration which determines tumor cell proliferation rate, the concentration of ECM large molecules which induced tumor cells chemotactic movement, and tumor surrounding tissue environment. These factors have a definite biological effect on tumor cells and are easily obtained from medical images or medical experience data. Based on the diffusion-convection-reaction equation, these small scale factors of cell level are respectively represented by the diffusion, the convection and reaction terms, and an overall tumor growth process in a large scale is shown in the iterative solution of equation. Meanwhile, the tumor cells are divide to two groups of proliferating cells and necrotic cells, thus a coupling model is proposed to reflect the interaction effect of these two cell groups in the actual dynamic tumor growth process. The simulation results show that the proposed model in this paper can show the overall tumor growth trend and the interaction between various factors and tumor cells, and is in line with the biological behavior of the actual tumor growth.

The model still stays at the stage of mathematical simulation, and the parameters are also dimensionless parameters, which need to be matched with the real tumor growth parameters. A key problem is how to quantify these parameters, such as proliferation rate and consumption rate, to make them have practical physical meanings and units. Furthermore, the angiogenesis [12] and tumor heterogeneity [13] are also have an important role in the process of tumor growth. These factors will be considered and modeled in our future work.

\section{Acknowledgments}

This work was financially supported by the Natural Science Foundation of Shandong Province (No. ZR2016HL42) and Science and Technology Development Program of Weifang City.

\section{References}

[1] T. Roose, S.J. Chapman, P.K. Maini, Mathematical models of avascular tumor growth, SIAM Rev. 49 (2007) 179-208.

[2] D. Alemani, F. Pappalardo, M. Pennisi and et al., Combining cellular automata and lattice Boltzmann method to model multiscale avascular tumor growth coupled with nutrient diffusion and immune competition, J. Immunol. Methods. 376 (2012) 55-68.

[3] M.W. Collins, Two-dimensional discrete mathematical model of tumor-induced angiogenesis, Appl. Math. Mech. 30 (2009) 455-462. 
[4] P. Gerlee, A.R.A. Anderson, An evolutionary hybrid cellular automaton model of solid tumor growth, J. Theor. Biol. 246 (2007) 583-603.

[5] J. LYU, S. Xu, W. Yao, Y. Zhou, Q. Long, Hybrid discrete-continuum model of tumor growth considering capillary points, Appl. Math. Mech. 34 (2013) 1237-1246.

[6] P. Macklin, S. McDougall, A.R.A. Anderson, and et al., Multiscale modelling and nonlinear simulation of vascular tumour growth, J. Math. Biol. 58 (2009) 765-798.

[7] K.A. Rejniak, A.R. A. Anderson, Hybrid models of tumor growth, WIREs Syst. Biol. Med. 3 (2011) 115-125.

[8] S.M. Wise, Three-dimensional multispecies nonlinear tumor growth-I model and numerical method, J. Theor. Biol. 253 (2008) 524-543.

[9] H.B. Frieboes, F. Jin, Y. Chuang, S.M. Wise, and et al., Three-dimensional multispecies nonlinear tumor growth-II tumor invasion and angiogenesis, J. Theor. Biol. 264 (2010) 1254-1278.

[10] Y. Cai, J. Wu, Q. Long, S. Xu, Z. Li, 3D numerical simulation of avascular tumour growth: effect of hypoxic micro-environment in host tissue, Appl. Math. Mech. 34 (2013) 1055-1068.

[11] M. Wu, H.B. Frieboes, M.A. Chaplain, S.R. McDougall, V. Cristini, J.S. Lowengrub, The effect of interstitial pressure on therapeutic agent transport: coupling with the tumor blood and lymphatic vascular systems, J. Theor. Biol. 355 (2014) 194-207.

[12] J. Meng, S. Dong, L. Tang and et al., A hybrid mathematical model of tumor-induced angiogenesis with blood perfusion, Tsinghua Sci. Technol. 19 (2014) 648-657.

[13] Z. Wang, J.D. Butner, R. Kerketta, V. Cristini and et al., Simulating cancer growth with multiscale agent-based modeling, Semin. Cancer Biol. 30 (2015) 70-78. 